This item was submitted to Loughborough's Research Repository by the author.

Items in Figshare are protected by copyright, with all rights reserved, unless otherwise indicated.

\title{
Dynamic responses of biological liquid to high intensity and sub-microsecond pulsed electric fields
}

PLEASE CITE THE PUBLISHED VERSION

PUBLISHER

(c) IEEE

VERSION

VoR (Version of Record)

\section{LICENCE}

CC BY-NC-ND 4.0

\section{REPOSITORY RECORD}

Chalise, Priya R., Bucur M. Novac, Ivor R. Smith, and Michael G. Kong. 2019. "Dynamic Responses of Biological Liquid to High Intensity and Sub-microsecond Pulsed Electric Fields". figshare.

https://hdl.handle.net/2134/5233. 
This item was submitted to Loughborough's Institutional Repository (https://dspace.lboro.ac.uk/) by the author and is made available under the following Creative Commons Licence conditions.

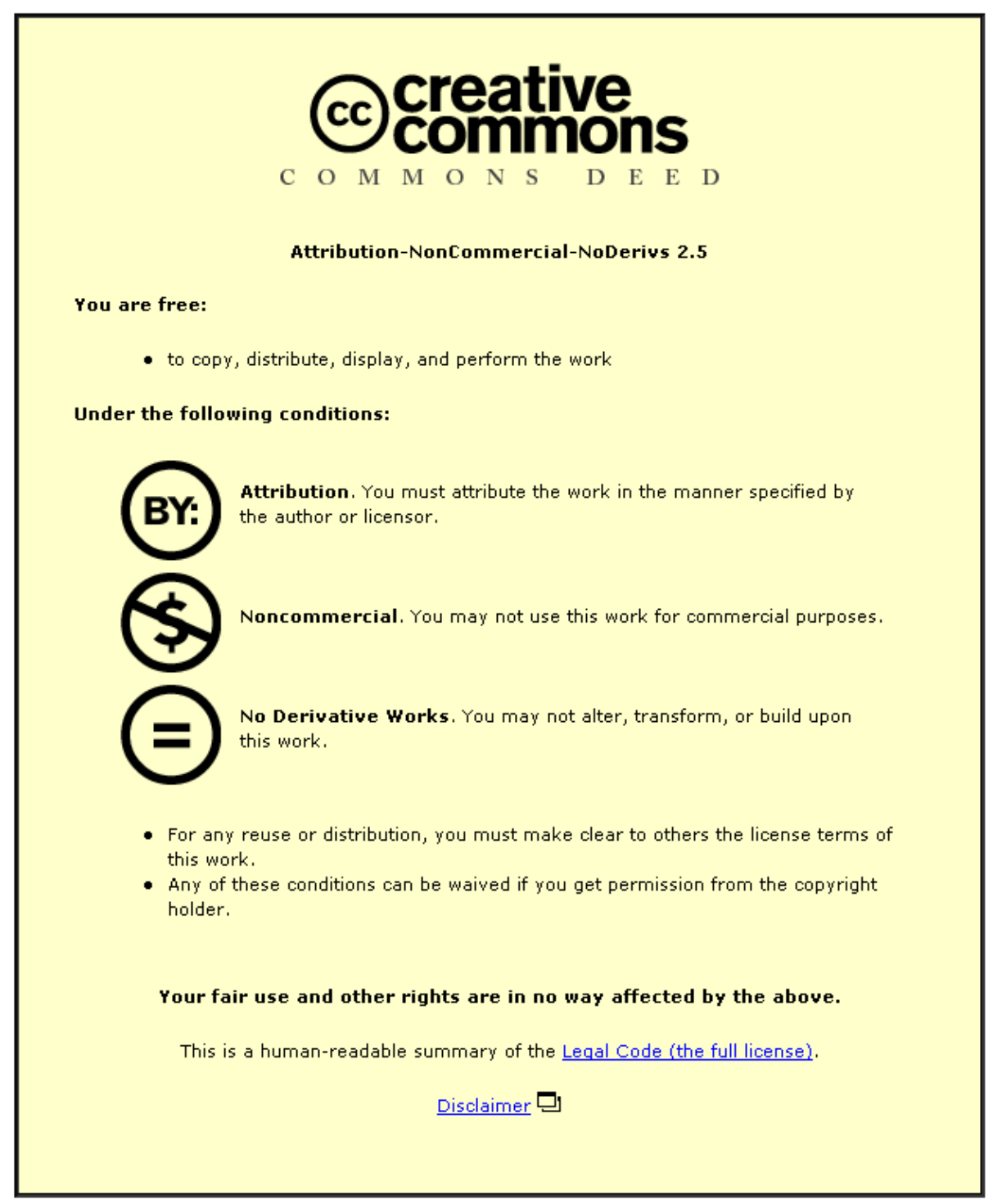

For the full text of this licence, please go to: http://creativecommons.org/licenses/by-nc-nd/2.5/ 


\title{
Dynamic Responses of Biological Liquid to High Intensity and Sub-Microsecond Pulsed Electric Fields
}

\author{
Priya R Chalise, Bucur M Novac, Ivor R Smith and Michael G Kong \\ Department of Electronic and Electrical Engineering, Loughborough University, Leicestershire LE11 3TU, UK.
}

\begin{abstract}
In bacterial decontamination of liquid food, the use of a high-intensity sub-microsecond pulse electric field offers a number of advantages, including increased breakdown strength of the liquid medium being treated. We present a measurement of temporal response of a sub-microsecond voltage pulse to show both pre-breakdown and breakdown conditions of a bulk biological liquid to establish a parametric range in which electrical breakdown can be avoided. The paper reports submicrosecond responses of current and voltage pulses, as well as dynamic impedance of the biological load, all of which are important in evaluating the performance of the pulsed electric technique in both cellular and bulk level.
\end{abstract}

\section{INTRODUCTION}

Pulse electric field (PEF) enabled applications such as bacterial decontamination of liquid food have attracted significant interest for many years [1]-[3]. One of the major drawbacks of this technique when operating in millisecond (ms) to microsecond ( $\mu$ s) scale, is an early electrical breakdown of liquids. Such undesirable breakdowns can generate active chemical species that would have a negative effect on sensorial and nutritional quality of the liquid food being treated. This is the reason why use of such $\mu$ sEF treatment is restricted to certain liquid foods whose electric resistance is high (or low conductivity) [2]. Such PEF technique is not suitable for most of the food products containing air bubbles, because the presence of bubbles causes partial discharges and early electrical breakdown [4]. But electrical resistance of most of the liquid foods are low (or high conductivity), and they also contains bubbles. One way to avoid the electrical breakdown in high voltage treatment of liquid foods is to separate them from high voltage electrode surfaces [5]; but in such system, energy transfer from the electrode to the liquid is low, due to a large potential drop near the electrode [6].

Recent technological advancement in the pulsed power technology has stimulated significant interest of high intensity and sub- $\mu$ s PEF technique, which is capable of elevating such electrical breakdowns to a much higher electrical field level. Moreover, if the pulse duration is in sub- $\mu$ s order, the PEF treatments are relatively insensitive to the bubbles [7]. Hence, it is important to establish a parametric space within which electrical breakdown is mitigated in sub- $\mu$ s pulses. In this paper, we present an experimental study to establish conditions of pre-breakdown and breakdown of a bulk biological load in a high voltage electric pulse of duration $700 \mathrm{~ns}$ and rise time 5 ns.

In order to understand how bacteria cells may respond dynamically to the sharp electrical pulses either as a bulk or on a molecular level, a direct measurement of pulsed parameters in largely fluctuating biological load may be a starting point. The direct measurement of amplitudes and durations of voltage and current pulses in biological loads are important because the increased electrical field increases inactivation efficiency at the cellular level [8], and the reduced pulse duration increases electrical efficiency [9] at the process level. The amplitudes and durations of the voltage and current pulses are significantly different from the output terminal of the PEF devices when they are subjected to a bulk biological load containing different liquid mediums. A pulsed current flowing into the bulk biological load not only provides important information on the conductivity of the bulk load, but also help to understand the interaction of the pulse current and biological cells in molecular level. But most PEF studies are confined only to the voltage measurement. One of the challenges associated with pulse current measurement in nanosecond (ns) to sub- $\mu$ s scale, is a lack of suitable commercial current monitors for large currents up to several kA. In this paper, we describe design and construction of a unique current monitoring system for high intensity fast pulse current measurement. We also present a simultaneous current and voltage pulses and extracted dynamic impedance of a representative biological load in single pulse event.

\section{EXPERIMENTAL DEVICE}

A high voltage trigger generator (TG-70, Maxwell Technologies Physics International, USA) was used as a PEF generator. A simplified schematic diagram of the generator and externally connected biological load is illustrated in Fig. 1. The pulse formation was achieved by charging a $0.1 \mu \mathrm{F}$ capacitor through $250 \Omega$ series resistor and discharging into three identical outputs following a triggering of spark gap switch. In the triggering circuit, a trigger amplifier (Pacific Atlantic Electronics Co., Model No. PT-55) was electrically connected 
to the trigger switch via two series $55 \Omega$ resistors. One of the TG output was connected to the biological load. A representative biological load consisting of cell suspension liquid, a phosphate buffered saline (PBS) at $\mathrm{pH} 7$, was placed in a commercially available cuvett ( $4 \mathrm{~mm}$ gap) attached between two plane metal electrodes. A typical voltage pulse across the biological load measured by a North Star PVM-6 probe with minimum rise time of $<4 \mathrm{~ns}$ (frequency response of $80 \mathrm{MHz}$ ) and oscilloscope (Tektronix TDS 5054B), is shown in Fig. 2. A typical voltage pulse is shown with the pulse duration of $700 \mathrm{~ns}$ at the rise time of $5 \mathrm{~ns}$ and amplitude of $12 \mathrm{kV}$ (electric field intensity of $30 \mathrm{kV} / \mathrm{cm}$ ).

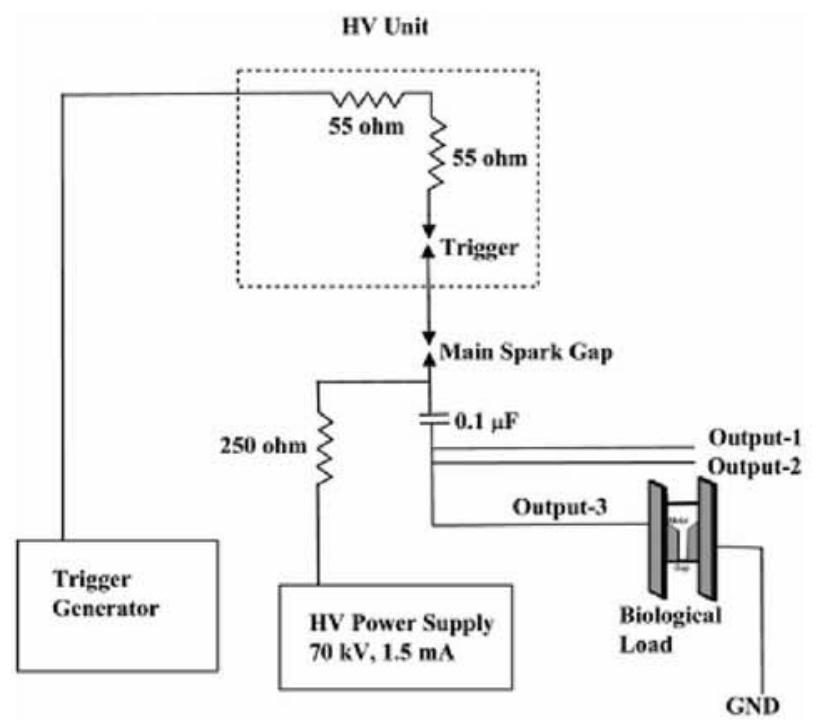

Fig. 1. Schematic diagram of experimental device.

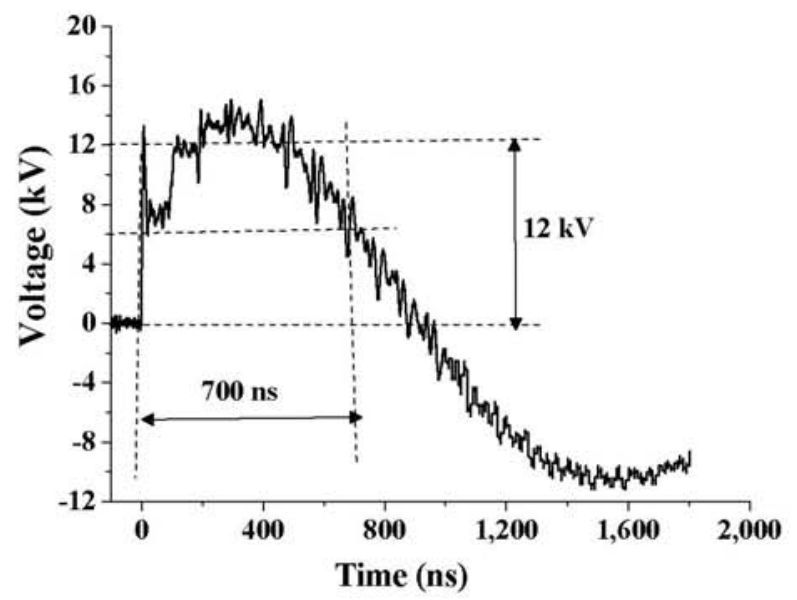

Fig. 2. A typical voltage pulse across a biological load.

\section{RESULTS AND DISCUSSION}

The breakdown phenomena in sub- $\mu$ s pulse up to ns scale is governed by mechanisms distinct from $\mathrm{ms}$ to $\mu$ s pulses. It is generally assumed that "thermal" processes causing bubble formations are associated with dielectric breakdown of liquids subjected to an electric field stress of $>1 \mu$ s duration, and "electronic" processes causing electron avalanche in liquid phase are associated with electric field stress of sub- $\mu$ s duration [10]. However, current understanding of electron avalanche mechanism is very limited, as most commonly used discharge investigations are predominately performed by $\mu \mathrm{s}$ pulses and consequently, the "bubble mechanism" dominates investigations. The use of sub- $\mu$ s pulses enables one to avoid the bubble formation in the bulk of the liquid and increase the breakdown strength. We expect that this dynamic breakdown data is also useful for those who are developing theoretical models of liquid breakdown in sub- $\mu$ s duration.

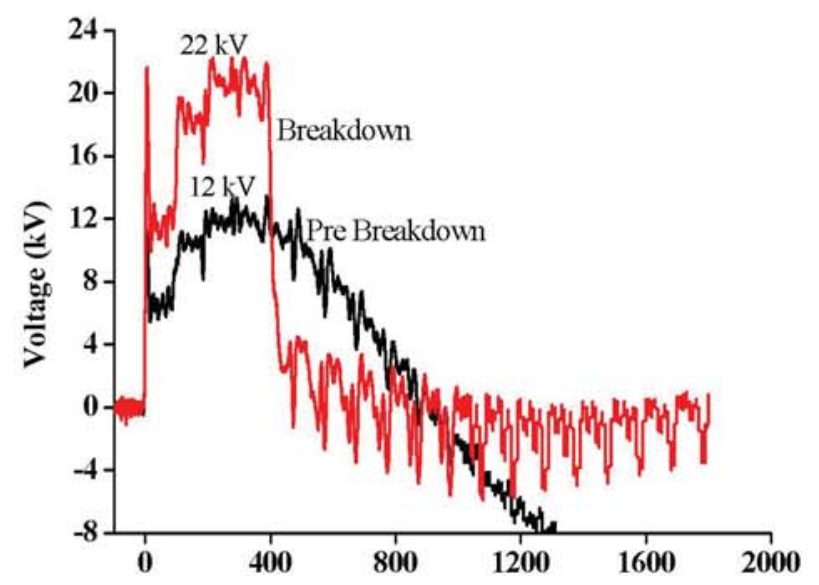

Fig. 3. Temporal responses of voltage pulse at pre breakdown and breakdown conditions.

The temporal responses of voltage pulse at both the pre breakdown and breakdown conditions are shown in Fig. 3. A pre breakdown voltage pulse shows the amplitude of $12 \mathrm{kV}(30$ $\mathrm{kV} / \mathrm{cm}$ ) and the duration of $700 \mathrm{~ns}$ at the rise time of $5 \mathrm{~ns}$ across the biological load. The capacitor charging voltage was $20 \mathrm{kV}$. As the voltage amplitude was increased to $22 \mathrm{kV}(55$ $\mathrm{kV} / \mathrm{cm}$ ) across the load, a complete breakdown of liquid was observed. The breakdown pulse showed similar rise time of 5 ns but a reduced pulsed duration. The $22 \mathrm{kV}$ pulse initially built up to attain the peak value where it remained in prebreakdown until the breakdown occurred at 400 ns. After the breakdown, the pulse amplitude suddenly decreased to the value very small compared to the applied voltage. The voltage oscillation of small magnitude can be observed, possibly due to the stray capacitance and inductance of the circuit. In the given electrode configuration and liquid medium, the breakdown may 
be avoided if the same PEF is applied at the pulse duration shorter than $400 \mathrm{~ns}$. If the pulse duration is further reduced, the electrical field intensity can be increased. This was observed in our nanosecond ( $32 \mathrm{~ns}$ ) pulse generator where the breakdown of PBS buffer in similar geometry was safely avoided in electrical field intensity up to $240 \mathrm{kV} / \mathrm{cm}$. Details of this nanosecond pulse experiment will be presented elsewhere.

We measured both the current and voltage response of high intensity (up to $25 \mathrm{kV}, 2.5 \mathrm{kA}$ pulse) in the pulse duration of $700 \mathrm{~ns}$ at the rise time of $5 \mathrm{~ns}$ across a low impedance biological load. The voltage diagnostic was relatively easy as a high voltage probe manufactured by North Star (model PVM 6) reliably measures pulse voltages up to $100 \mathrm{kV}$ with frequency response of $80 \mathrm{MHz}$. A special arrangement was needed to detect the current pulse because no commercial current probe capable of detecting such $(<5$ ns and $2.5 \mathrm{kA})$ pulses is available.

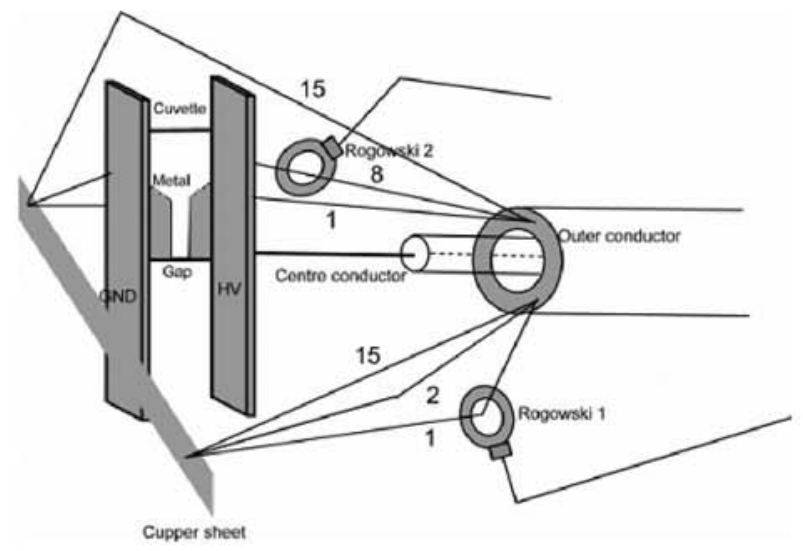

Fig. 4. Schematic arrangement of current monitoring system.

We have modified a current monitoring system by dividing the returning current into large number of low inductance wires. The schematic arrangement of such a current monitoring system is shown in Fig. 4. The current signals are monitored at two extreme ends of the wires by using a commercially available Pearson current monitor (2887). Two Pearson monitors, placed at two extreme ends of the wires to check the uniformity of current distribution, are capable of detecting usable rise time of $2 \mathrm{~ns}$ at the peak current of $100 \mathrm{~A}$. For the current monitoring, the high voltage coaxial cable output of the PEF device (TG-70) has been reconfigured before connecting to the electrodes. The inner conductor of the coaxial cable was connected to one end of high voltage electrode connecting the cuvette. The outer conductor carrying the returning current and distributing it into 30 parallel paths was connected to high voltage electrodes through thin sheets of copper. Together with a high voltage probe, the modified current monitor was used to detect the dynamic response of current and voltage pulse as well as dynamic impedance of the biological load.

Fig. 5 shows temporal characteristics of current and voltage pulses, which were simultaneously measured across the biological load. The voltage pulse is the same as before, with the amplitude of $12.5 \mathrm{kV}$ (electric field intensity, $30 \mathrm{kV} / \mathrm{cm}$ ) and the duration of $700 \mathrm{~ns}$ at the rise time of $5 \mathrm{~ns}$. The current pulse is almost in the phase and similar pulse duration with the voltage pulse, but the peak amplitude is $2.4 \mathrm{kA}$. This indicates a large amount of current passing into the cell suspension. The bulk current is distributed in a number of biological load components such as individual cells, suspension liquid, electrodes, and interfaces. Almost similar phase of voltage and current pulse indicates that the capacitive effect of the bulk biological load is not significantly influencing the pulse parameters.

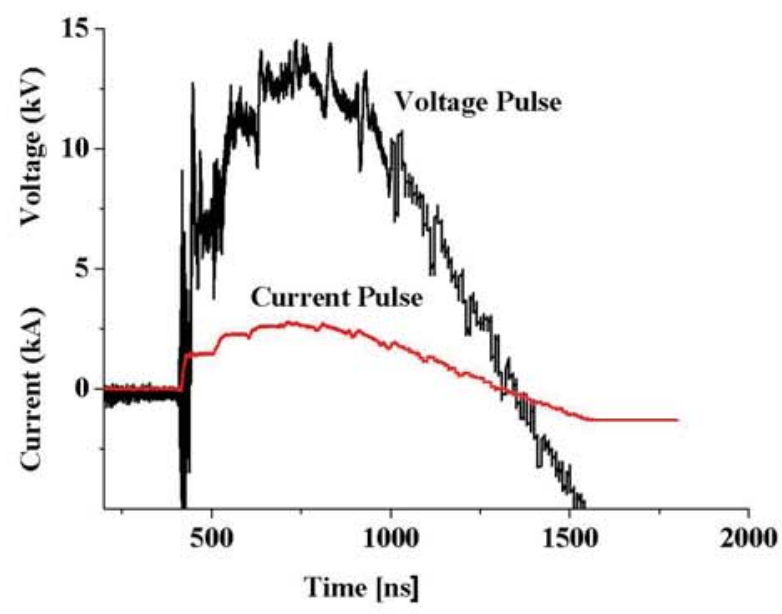

Fig. 5. Temporal characteristics of current and voltage pulses.

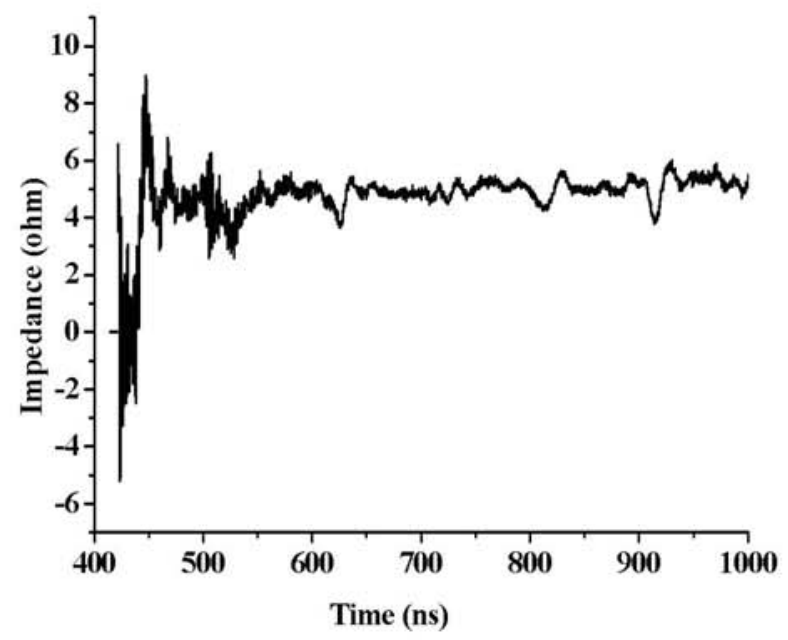

Fig. 6. Dynamic impedance of the bulk biological load.

The dynamic impedance of the bulk biological load extracted from the current and voltage waveform is shown in Fig. 6 . The bulk load impedance reaches $8 \Omega$ in a time duration up to 
approximately $100 \mathrm{~ns}$, hen maintains almost a constant level of $5.8 \Omega$ for the rest of the pulse period. The bulk load impedance of a sub $\mu$ s pulse is higher than the measured DC impedance value (3.2) $\Omega$.

\section{SUMMARY}

We have presented a temporal response of a sub- $\mu$ s voltage pulse to show both pre-breakdown and breakdown conditions of a bulk biological load. For example, PBS at $\mathrm{pH} 7$ can withstand $55 \mathrm{kV} / \mathrm{cm}$ for about $400 \mathrm{~ns}$ before irreversible breakdown is ignited. A sub- $\mu$ s temporal evolution of current and voltage pulses as well as dynamic impedance of bulk biological load has been measured using a self-developed fast pulse diagnostic technique. It is observed that the impedance of the biological load fluctuates initially up to $100 \mathrm{~ns}$, then maintains almost a constant level of $5.8 \mathrm{ohm}$ for the rest of the pulse period. The results represent a useful starting point to understand how bacterial cells may respond dynamically to sharp electrical pulses, either as a bulk or on a molecular level. These are just a few preliminary results but a detailed study on dynamic response of breakdown and impedances of different biological loads is planned for the future.

\section{ACKNOWLEDGMENT}

This research work is funded by Engineering and Physical Sciences Research Council (EPSRC) of the United Kingdom.

\section{REFERENCES}

[1] B. L. Qin et al.,"Inactivating microorganism using a pulsed electric field continuous treatment system," IEEE Trans. Industrial Appl., vol. 34, no.1, pp. 43-49,1998.

[2] P. C. Wouters "Inactivation of Microorganisms with Pulsed Electric Fields: Potential for Food Preservation," Food Biotechnology, vol. 11, pp. 193-223, 1997.

[3] S. Bendicho, A. Espachs, J. Arantegui, and O. Martin, "Effect of high intensity pulse electric fields and heat treatments on vitamins of milk," Journal of Dairy Research, vol. 69, pp. 113-123, 2002.

[4] Report- "Kinetics of Microbial inactivation for alternative food processing technologies- pulsed electri fields," U.S. Food and Drug Administration, Centre for Food Safety and Applied Nutritions, 2 June 2000.

[5] Mazurek, P. Lubicki and Z. Staroniewicz, "Effect of short HV pulses on bacteria and fungi," IEEE Trans. on Dielectric and Electrical Insulation, vol. 2 , no. 3, pp. 418-425, 1997

[6] S. H. Jayaram, "Sterilization of liquid foods by pulsed electric fields,", IEEE Electrical Insulation magazine, vol. 16, no.6, pp 17-25, 2000.

[7] H. M. Jones and E. E. Kunhardt, " development of pulsed dielectric breakdown in liquids," J. Phys. D: Appl. Phys., vol. 28, pp. 178-188, 1995.

[8] P. C. Wouters, I. Alverge and J. Raso, "Critical factor determining inactivation kinetics by pulsed electric field food processing," Trend in Food Science and Technology, vol.12, pp.112-121, 2001.

[9] K. H. Schoenbach et al., "Bacterial decontamination of liquid with pulse electric fields", IEEE Trans. on Dielectrics and Electrical Insulation, vol. 7, no. 5, pp. 637-645, 2000.

[10] A. A. Belevtsev and V. V. Markovets, "Nanosecond breakdown in liquid nitrogen," J. Phys. D: Appl. Phys., vol. 34, pp. L52-L56, 2001. 